\section{Ecuaciones para la evaluación de la composición corporal en niños y adolescentes}

\author{
Equations for the evaluation of body composition \\ in children and adolescents
}

\section{Equações para a avaliação da composição corporal em crianças e adolescentes}

Cristian Curilem-Gatica 1

Fernando Rodríguez-Rodríguez 1

Atilio Almagià-Flores 1

Tuillang Yuing-Farías 1

Francisco José Berral-de-la-Rosa 2

\title{
Resumen
}

El uso del índice de masa corporal (IMC) es el más masivo a nivel mundial y a pesar de existir recomendaciones para su interpretación en relación al sexo, edad y raza, normalmente se estandariza su entendimiento, aumentando el error en el resultado y en la clasificación del estado nutricional. El uso de la composición corporal entrega información de mayor relevancia que el IMC, siendo la masa grasa y la masa muscular las principales. Esta comunicación presenta una revisión de las ecuaciones existentes y propone aquellas más simples y con menor error de estimación para ser usadas como una herramienta que reemplace o complemente al IMC, favoreciendo una mejor comprensión e interpretación del estado nutricional y nivel de actividad física.

Índice de Masa Corporal; Composición Corporal; Niño; Adolescente

1 Pontificia Universidad Católica de Valparaíso Valparaíso, Chile. 2 Universidad Pablo de Olavide, Sevilla, España.

\author{
Correspondencia \\ C. Cuilem-Gatica \\ Pontificia Universidad \\ Católica de Valparaíso. \\ Av. Universidad 330 \\ Curauma, Valparaíso \\ 2340000, Chile. \\ c.curilem.g@gmail.com
}




\section{Índices para la evaluación de escolares}

El índice de masa corporal (IMC) es un indicador que se utiliza ampliamente para diagnosticar el estado nutricional de los escolares. En la actualidad, existen varios estándares para este índice en niños y adolescentes realizados en diferentes países, que tienen diferencias significativas para igual percentil 1. Cole et al. 2 señalan que los puntos de corte para la clasificación del IMC varían entre países, considerando un rango (Z-score) para diversas poblaciones, en tanto es necesario para una mejor precisión en la medición considerar el sexo, la edad y el país.

El criterio actual, para el diagnóstico de la obesidad en niños y adolescentes, es más estadístico que biológico, donde normalmente se aplica un estándar universal de distribución por percentiles, o quienes con menos certeza usan los valores equivalentes al índice de masa corporal de riesgo y obesidad del adulto. Asimismo, este índice no refleja fielmente los cambios de la composición corporal que ocurren durante la adolescencia, por lo que hacer una valoración solamente con este índice es insuficiente para diagnosticar sobrepeso, o un estado nutricional de manera correcta, siendo muy importante detallar los componentes corporales ${ }^{3}$. Una de las principales limitaciones del IMC corresponde a que su incremento podría deberse al aumento de la masa magra, considerando, además, que su relación con el tejido graso es relativo y que varía de acuerdo con la edad, el sexo, el tipo de población y el grado de madurez sexual. Se ha establecido que la distribución del tejido graso en la región abdominal, más específicamente el tejido perivisceral (mesenterios y omentos), se asocia con mayor riesgo de enfermedad cardiovascular, diabetes mellitus tipo II y cáncer, entre otras enfermedades, sin necesariamente tener un IMC de sobrepeso u obeso. Se trata de un aspecto que merece especial consideración, pues se ha observado que el tejido graso central abdominal se ha incrementado mucho más que el tejido graso total en niños y el IMC sería un indicador menos sensible a esta diferencia ${ }^{4}$.

La aplicación práctica del perímetro de cintura y su asociación con factores de riesgo cardiovascular, además de la correlación fuerte con el área grasa visceral, medida por tomografía computarizada, son características que lo transformaron en el indicador de grasa abdominal más utilizado ${ }^{5}$. A pesar de que el perímetro de cintura se ha difundido extensamente, hay descripciones diferentes de los lugares anatómicos donde se debe hacer la medición y, consecuentemente, existe una ausencia de consenso entre los investigadores y protocolos publicados, lo que puede generar conflictos en el momento de evaluar en la comparación de los resultados de diferentes trabajos. Respecto a la Sociedad Internacional de Avances en Kineantropometría (ISAK), se indica que la medición del perímetro de cintura mínima debe realizarse mediante una cinta inextensible de acero flexible calibrada en centímetros, con gradaciones en milímetros, en el nivel del punto más estrecho entre la 12a costilla y la cresta ilíaca si la zona más estrecha no es aparente, entonces la lectura se realiza en el punto medio entre estas dos marcas.

Actualmente, se ha incorporado el índice cintura/estatura, como una sencilla medida antropométrica complementaria, que presenta una buena correlación con los indicadores de riesgo cardiovascular en niños y adolescentes. En niños y adolescentes, la literatura reciente destaca el uso de un punto de corte de $\geq 0,55$ y que su incremento se correlaciona con el aumento de factores de riesgo cardiovasculares y metabólicos adversos, independiente de la edad, el sexo y el origen étnico.

\section{Composición corporal}

En la actualidad existen diversas metodologías para determinar el componente muscular y graso en niños y adolescentes, favoreciendo el seguimiento, evaluación e investigación de las medidas preventivas en niños y adolescentes. Entre estas metodologías se encuentran las que determinan la densidad corporal (Tabla 1) y, a partir de esta variable, estiman la masa grasa, utilizando principalmente los pliegues cutáneos como principal determinante. Es importante destacar que estas ecuaciones son doblemente indirectas, siendo el error de estimación, su principal limitante, ya que al error de la fórmula para obtener densidad, se suma el error de la estimación para obtener el porcentaje graso ${ }^{6}$. Por otra parte, el músculo como órgano endocrino, tiene más relevancia y se relaciona mejor con condicionantes de la salud que la masa grasa, por lo que su determinación debe ser considerada sin dudas. Las ecuaciones determinan principalmente el área muscular braquial (Tabla 2), sin considerar los miembros inferiores, desestimando estas regiones, así como la funcionalidad y relación con el nivel de actividad física en niños y adolescentes.

Al revisar las diferentes metodologías, proponemos el uso de las ecuaciones de Poortmans et al. ${ }^{7}$ para cuantificar el componente muscular, para niños y niñas de entre 7 y 16 años de edad, validado por DEXA y con un valor de $\mathrm{R}^{2}=0,96 \mathrm{y}$ un valor de $\mathrm{p}<0,001$. Del mismo modo, la ecuación de Slaughter et al. 8, validada igualmente 
Tabla 1

Ecuaciones para estimar el componente graso.

\begin{tabular}{|c|c|c|}
\hline Autor (año) & Edad (años) & Ecuaciones \\
\hline \multirow[t]{2}{*}{ Brook 11 (1971) } & $1-11$ & (Mujeres) D = 1,2063-0,0999 (LOG $\Sigma 4$ pliegues) \\
\hline & & (Hombres) D = 1,1690-0,0788 (LOG $\Sigma 4$ pliegues) \\
\hline Durnin \& Rahaman 12 & $13-15,9$ & (Mujeres 13-15,9 años) $D=1,1369-0,0598$ (LOG $\Sigma 4$ pliegues) \\
\hline (1967) & & (Hombres 13-15,9 años) $D=1,1533-0,0643$ (LOG $\Sigma 4$ pliegues) \\
\hline Durnin \& Womersley 13 & $16-19,9$ & (Mujeres 16-19,9 años) D = 1,1549-0,0678 (LOG $\Sigma 4$ pliegues) \\
\hline (1974) & & (Hombres 16-19,9 años) D = 1,162-0,063 (LOG $\Sigma 4$ pliegues) \\
\hline \multirow{2}{*}{ Johnston et al. 14 (1988) } & $8-14$ & (Mujeres) $\mathrm{D}=1,144-0,06$ (LOG $\Sigma 4$ pliegues) \\
\hline & & (Hombres) $\mathrm{D}=1,166-0,07$ (LOG $\Sigma 4$ pliegues) \\
\hline Deurenberg et al. 15 & Pubertad mujer & (Mujeres pubertad) $D=1,1074-0,0504$ (LOG $\Sigma 4$ pliegues) $+1,6$ (edad 103) \\
\hline \multirow[t]{4}{*}{ (1990) } & Pubertad hombre & (Hombres pubertad) $\mathrm{D}=1,0555-0,0352$ (LOG $\Sigma 4$ pliegues) $+3,8($ edad 103) \\
\hline & Postpubertad mujer & $\mathrm{D}=1,183-0,0813$ (LOG $\Sigma 4$ pliegues) \\
\hline & Postpubertad & $\mathrm{D}=1,1324-0,0429$ (LOG $\Sigma 4$ pliegues) \\
\hline & hombre & \\
\hline \multirow[t]{2}{*}{ Sarría et al. 16 (1998) } & $11-16,9$ & (Hombres 11-13,9 años) $D=1,1516-0,0658$ (LOG $\Sigma 4$ pliegues) \\
\hline & & (Hombres 14-16,9 años) D = 1,169-0,0693 (LOG $\Sigma 4$ pliegues) \\
\hline Wilmore \& Behnke 17 & Mujeres jóvenes & (Mujeres) $D=1,06234-0,00068$ pliegue subescapular - 0,00039 pliegue tricipital - 0,00025 pliegue \\
\hline
\end{tabular}

D: densidad (kg/l); $\Sigma 4$ pliegues: bíceps + tríceps + subescapular + suprailíaco (mm).

\section{Tabla 2}

Ecuaciones para estimar el componente muscular.

\begin{tabular}{|c|c|}
\hline Autor (año) & Ecuación \\
\hline Frisancho 18 (1981) & Área muscular brazo $\left(\mathrm{mm}^{2}\right)=($ perímetro brazo $-\pi$ pliegue tricipital2 $) / 4 \pi$ \\
\hline \multirow[t]{5}{*}{ Heymsfield et al. 19 (1982) } & Área muscular braquial $\left(\mathrm{cm}^{2}\right)=([$ perímetro brazo relajado $-(\pi$ * pliegue tricipital $)] 2 / 4 * \pi)-10$ \\
\hline & (Hombres) \\
\hline & Área muscular braquial $=([$ perímetro brazo relajado $-(\pi *$ pliegue tricipital $)] 2 / 4 * \pi)-6,5$ \\
\hline & (Mujeres) \\
\hline & Masa muscular $(\mathrm{kg})=$ estatura $(\mathrm{cm}) *[0,0264+(0,0029$ * Área muscular braquial $)]$ \\
\hline \multirow[t]{2}{*}{ Lee et al. 20 (2000) } & Masa muscular $(\mathrm{kg})=0,226 \times$ peso $+13 \times$ estatura $\times(-0,089) \times$ edad $+6,3 \times$ sexo + raza -11 \\
\hline & Sexo $(1=$ masculino $)(0=$ femenino $) ;$ raza $(-1,6$ asiático $)(1,9$ afroamericano $)(0=$ blanco $)$ \\
\hline
\end{tabular}

por DEXA, es una excelente alternativa para el componente graso, debido a que está diseñada para niños y adolescentes de 8 a 18 años, con un error de estimación del 3,8\%, además presenta ecuaciones con los pliegues del tríceps y subescapular, para sujetos blancos y negros (Tabla 3). Ambos métodos son propuestos de- bido a su práctica aplicación y alta correlación en niños y adolescentes 9, ambos métodos evalúan los miembros, fundamentales para la funcionalidad y motricidad de los jóvenes; además son metodologías estandarizadas, válidas y confiables 10 . 
Curilem-Gatica $C$ et al.

Tabla 3

Ecuaciones antropométricas para estimar masa grasa y masa muscular en adolescentes.

Ecuación

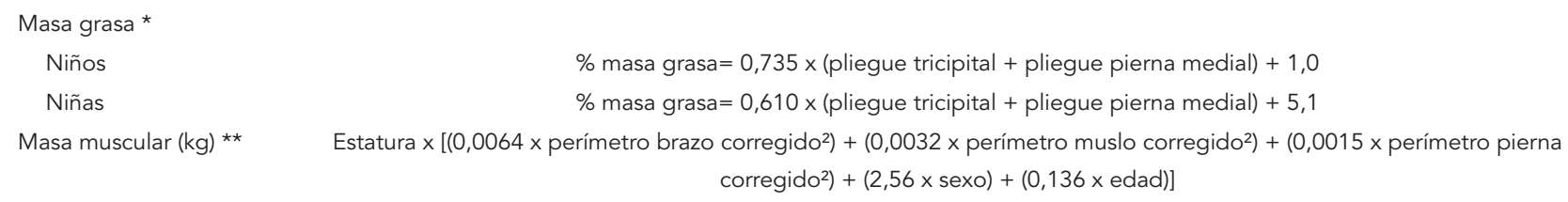

Perímetro brazo corregido: perímetro brazo relajado - (pliegue tríceps/10); perímetro muslo corregido: perímetro muslo medio - (pliegue muslo anterior/10); perímetro pierna corregido: perímetro pierna - (pliegue pierna/10).

* Slaughter et al. 8 .

** Poortmans et al. 7.

Nota: perímetros en centimetros $(\mathrm{cm})$; estatura en metros $(\mathrm{m})$; pliegue en milimetros $(\mathrm{mm})$; mujer: 0 ; hombre: 1 ; edad en años.

\section{Colaboradores}

C. Curilem-Gatica participó em la concepción y proyecto, análisis e interpretación de los datos, redacción y revisión crítica relevante del contenido intelectual del artículo y aprobación final de la versión que debe ser publicada. F. Rodríguez-Rodríguez colaboró en el análisis e interpretación de los datos, revisión crítica relevante del contenido intelectual y aprobación final de la versión que debe ser publicada. A. Almagià-Flores, T. Yuing-Farías, F. J. Berral-de-la-Rosa participaron del análisis e interpretación de los datos, em la revisión crítica relevante del contenido intelectual de artículo y aprobación final de la versión que debe ser publicada. Los autores son responsables de todos los aspectos del trabajo, garantizando la exactitud e integridad de cualquier parte de la obra.

\section{Agradecimientos}

A la Dirección de Investigación e Innovación de la Pontificia Universidad Católica de Valparaíso, por su constante apoyo. 


\section{Referencias}

1. Gotthelf S, Mendes M. Hipertensión arterial y su asociación con variables antropométricas en adolescentes escolarizados de la ciudad de Salta (Argentina). Rev Fed Argent Cardiol 2012; 41:96-102.

2. Cole TJ, Flegal KM, Nicholls D, Jackson AA. Body mass index cut offs to define thinness in children and adolescents: international survey. BMJ 2007; 335:166-7.

3. Díaz J, Espinoza-Navarro O. Determinación del porcentaje de masa grasa, según mediciones de perímetros corporales, peso y talla: un estudio de validación. Int J Morphol 2012; 30:1604-10.

4. Moreno LA, Moliner-Urdiales D, Ruiz JR, Mesana MI, Vicente-Rodríguez G, Rodríguez G, et al. Five year trends on total and abdominal adiposity in Spanish adolescents. Nutri Hosp 2012; 27:731-8.

5. Castro TG, Barufaldi LA, Schlüssel MM, Conde WL, Leite MS, Schuch I. Waist circumference and waist circumference to height ratios of Kaingang indigenous adolescents from the state of Rio Grande do Sul, Brazil. Cad Saúde Pública 2012; 28:2053-62.

6. Barbosa L, Cardoso O, Ribeiro R. Anthropometric and body composition parameters to predict body fat percentage and lipid profile in schoolchildren. Rev Paul Pediatr 2012; 30:520-8.

7. Poortmans J, Boisseau N, Moraine J, MorenoReyes R, Goldman S. Estimation of total-body skeletal muscle mass in children and adolescents. Med Sci Sports Exerc 2005; 37:316-23.

8. Slaughter MH, Lohman TG, Boileau RA, Horswill CA, Stillman RJ, Van Loan MD, et al. Skinfold equations for estimation of body fatness in children and youth. Hum Biol 1988; 60:709-23.

9. Rodríguez G, Moreno LA, Blay MG, Blay VA, Fleta G, Sarría A, et al. Body fat measurement in adolescents: comparison of skinfold thickness equations with dual-energy X-ray absorptiometry. Eur J Clin Nutr 2005; 59:1158-66.

10. Alvero Cruz JR, Cabañas Armesilla MD, Herrero de Lucas A, Martínez Riaga L, Moreno Pascual C, Porta Manzañido J, et al. Protocolo de valoración de la composición corporal para el reconocimiento médico-deportivo. Documento de consenso del grupo español de cineantropometría de la Federación Española de Medicina del Deporte. Arch Med Deporte 2009; 26:166-79.
11. Brook C. Determination of body composition of children from skinfold measurements. Arch Dis Child 1971; 46:182-6.

12. Durnin J, Rahaman M. The assessment of the amount of fat in the human body from meaurements of skinfold thickness. Br J Nutr 1967; 21: 681-90.

13. Durnin J, Womersley J. Body fat assessed from total body density and its estimation from skinfold thickness: measurements on 481 men and women aged from 16 to 72 years. Br J Nutr 1974; 32:77-98.

14. Johnston J, Leong M, Checkland E, Zuberbuhler P, Conger P, Quinney H. Body fat assessed from body density and estimated from skinfold thickness in normal children and children with cystic fibrosis. Am J Clin Nutr 1988; 48:1362-6.

15. Deurenberg P, Pieters J, Hautvast J. The assessment of the body fat percentage by skinfold thickness measurements in childhood and young adolescence. Br J Nutr 1990; 63:293-303.

16. Sarría A, García-Llop LA, Moreno LA, Fleta J, Morellón MP, Bueno M. Skinfold thickness measurements are better predictors of body fat percentage than body mass index in male Spanish children and adolescents. Eur J Clin Nutr 1998; 52:573-6.

17. Wilmore J, Behnke A. An anthropometric estimation of body density and lean body weight in young women. J Appl Physiol 1969; 27:25-31.

18. Frisancho A. New norms of upper limb fat and muscle areas for assessment of nutritional status. Am J Clin Nutr 1981; 34:2540-5.

19. Heymsfield S, MacManus C, Smith J, Stevens V, Nixon D. Anthrometric measurement of muscle mass: revised equations for calculating bone-free arm muscle area. Am J Clin Nutr 1982; 36:680-90.

20. Lee R, Wang Z, Heo M, Ross R, Janssen I, Heymsfield S. Total-body skeletal muscle mass: development and cross - validation of anthropometric prediction models. Am J Clin Nutr 2000; 72:796803. 


\section{Abstract}

Body mass index (BMI) is widely used in the world, despite caveats concerning its interpretation in relation to gender, age, and race. BMI reading is normally standardized, potentially producing error in the results and classification of nutritional status. Body composition provides more relevant information than BMI: fat mass and lean mass are the main indices. This paper reviews existing equations and proposes the simplest ones and those with the lowest estimation error to replace or complement BMI and improve interpretation of nutritional status and physical activity.

Body Mass Index; Body Composition; Child; Adolescent

\section{Resumo}

O índice de massa corporal (IMC) é amplamente utilizado ao nível mundial, apesar de existir recomendações para sua interpretação em relação ao sexo, idade e raça; normalmente o seu entendimento é padronizado, aumentando o erro no resultado e na classificação do estado nutricional. O uso da composição corpora oferece informações de maior relevância que o IMC sendo a massa gorda e massa muscular as principais. Esta comunicação apresenta uma revisão das equações existentes e propõe aquelas mais simples, e com menor erro de estimativa, para ser usadas como uma ferramenta que substitua ou complemente ao IMC, favorecendo uma melhor compreensão e interpretação do estado nutricional e nível da atividade física.

Índice de Massa Corporal; Composição Corporal; Criança; Adolescente
Recibido el 30/Dic/2014

Versión final presentada el 04/Sep/2015

Aprobado el 12/May/2016 